\title{
Geospatial intelligence about urban areas using SAR
}

\author{
A.C. van den Broek, R.J. Dekker, TNO Defence, Security and Safety, P.O. Box 96864, 2509 JG, \\ The Hague, The Netherlands, bert.vandenbroek@tno.nl
}

\begin{abstract}
Radar satellites are important for geospatial intelligence about urban areas and urban situational awareness, since these satellites can collect data at day and night and independently of weather conditions ensuring that the information can be obtained at regular intervals and in time. For this purpose we have applied change detection techniques developed at TNO to Radarsat I fine beam imagery of various dates to find changes in Baghdad during and after the war in 2003.

A drawback of SAR imagery is the poor ability to recognize the detected changes in the scene. In this paper we present a workflow for the characterization and classification of changes detected in SAR imagery. We show that these changes can be characterized using complementary data and context information. For this purpose we have used a digital surface model from Ikonos stereo imagery that contains building heights. We also have used so-called temporal features extracted from a multi-temporal data-set of Radarsat data to select the changes and to detect activity between 2003 and 2007, which has been classified with high-resolution optical data.
\end{abstract}

Keywords: Change detection, classification, synthetic aperture radar, geospatial intelligence, urban area

\section{INTRODUCTION}

Situational awareness and accurate up-to-date intelligence are keywords in present day urban area security and military operations on urbanized terrain. For surveillance in general and for gathering up-to-date (geospatial) intelligence with respect to new and/or damaged infrastructure, removal and storage of goods in cities, detection of changes using satellite data is an efficient approach.

In order to detect changes regular monitoring is required with time intervals, which critically depend on the phenomenon that has to be seen. The possible monitoring intervals depend on the number of satellites and the revisit time of the satellites. For a single satellite revisit times are in the order of weeks to several days, while daily monitoring requires several satellites. If short monitoring intervals are required without any observation gaps SAR imaging is indispensable due to its unparalleled all-time and all-weather capability. In the near future radar satellites with resolutions up to one meter, such as Terrasar- $\mathrm{X}$ and Cosmo-skymed will be operational so that accurate monitoring of urban areas with radar satellites becomes feasible.

Detection of a change is not enough since also the changes need to be characterized. For this purpose higher resolution is beneficial, but even with high-resolution SAR imaging recognition and identification of objects in the image is a difficult task. In this paper we present a workflow how to characterize changes, using multi-temporal data-sets in combination with high resolution optical satellite imagery and detailed geographical information. The workflow will be illustrated with a case study about monitoring Baghdad during Operation Iraqi Freedom in 2003 and the years after using multitemporal Radarsat fine beam imagery.

The paper is organized as follows. First we introduce in section 2 a general workflow for urban situational awareness on basis of radar satellite imagery and complementary data. In section 3 we give an overview of the change detection techniques used and apply these to the data. In section 4 we discuss change categorization. In section 5 we classify the changes and discuss the results. Finally in section 6 we present conclusions.

The study was performed in the framework of the NATO/SET working group on High Resolution SAR-Systems for Geospatial Intelligence (SET102) and the EU network of excellence on Global Monitoring of Security and Stability (GMOSS).

Remote Sensing for Environmental Monitoring, GIS Applications, and Geology VII, edited by Manfred Ehlers, Ulrich Michel, Proc. of SPIE Vol. 6749, 67490T, (2007) · 0277-786X/07/\$18 · doi: 10.1117/12.738486 


\section{WORKFLOW FOR SITUATIONAL AWARENESS}

We present here a workflow for obtaining accurate and up-to-date geospatial intelligence for the purpose of situational awareness. This workflow is more generally applicable, but we will use it here for an urban context. The workflow is depicted in figure 2.1. We identify three main issues. 1) Detection of changes with radar satellites, false alarm reduction and selection. 2) Obtaining up-to-date geographical information for the urban area using recent maps, GIS data, highresolution multi-spectral imagery, and a high-resolution digital surface model, for example obtained from high-resolution optical satellite stereo imagery. 3) Up-to-date description of the situation by detecting and characterizing recent changes using radar imagery combined with other information sources.

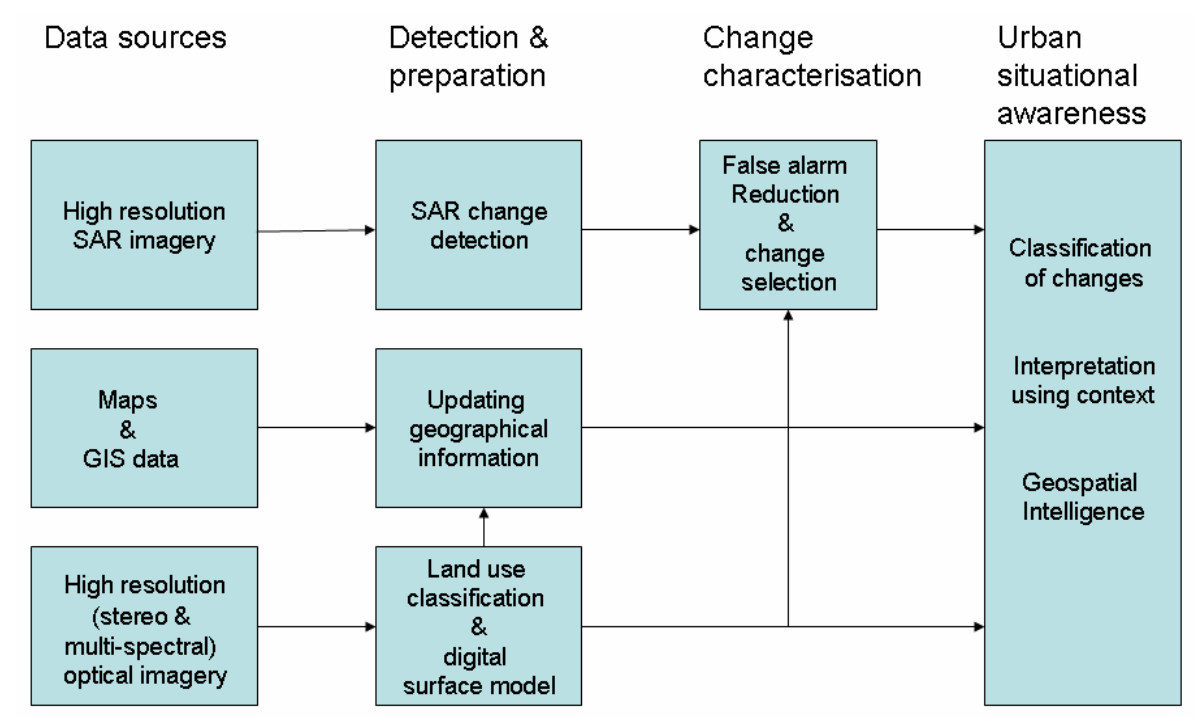

Figure 2.1. Workflow for urban situational awareness using high resolution SAR imagery

In the following sections we discuss the main steps of the workflow: SAR change detection and change characterization, followed by a discussion of urban situational awareness.

\section{SAR CHANGE DETECTION}

Change detection can be done using pairs of images with a certain time interval for example to look for changes due to a certain event with one image before and one image after. Another approach is to use multi-date data-sets, which are appropriate to monitor the multi-temporal behavior at locations in the scene. For change detection speckle noise present in SAR imagery is a problem since the speckle varies independently for images of different dates, which give rise to unwanted false alarms. We discuss here two techniques, which also handle the speckle problem. One technique is applicable to pairs of images and one to multi-date data-sets.

\subsection{Change detection on pairs of SAR images}

At TNO we have developed and implemented a change detection algorithm for efficiently co-registering imagery and extracting areas of change. The tool can be used for both optical as well as radar imagery. For radar imagery a dedicated filtering mechanism suppresses speckle in order to reduce unwanted false alarms. The change detection tool comprises the following processing steps: 1) Co-registration. For change detection it is essential that the images are perfectly aligned, i.e. co-registered accurately enough, since otherwise false changes will be introduced. In this step small local displacements between two successive images are detected by correlation and then removed. 2) Filtering. In this step the ratio is taken of the powers values in two successive images. Filtering of the ratio is performed using appropriate templates which preserve points, lines and edges (Dekker, 2005). 3) Threshold. A next step is to apply a threshold to the filtered image ratio to select the pixels, which can be attributed to changes. 4) Initial false alarm reduction. As a following step adjacent pixels are grouped and isolated pixels can be rejected, so that as a final result clusters of pixels 
are obtained which represent areas of change. For these clusters features can be derived such as dimension, mean intensity, texture etc. It is possible to use a land use map to discard changes in certain regions. Additional false alarm reduction will be discussed in the following section. 5) Raster to vector conversion. Finally the clusters are converted into polygons with the features as attributes. These attributes can later be used for further selection of the changes.

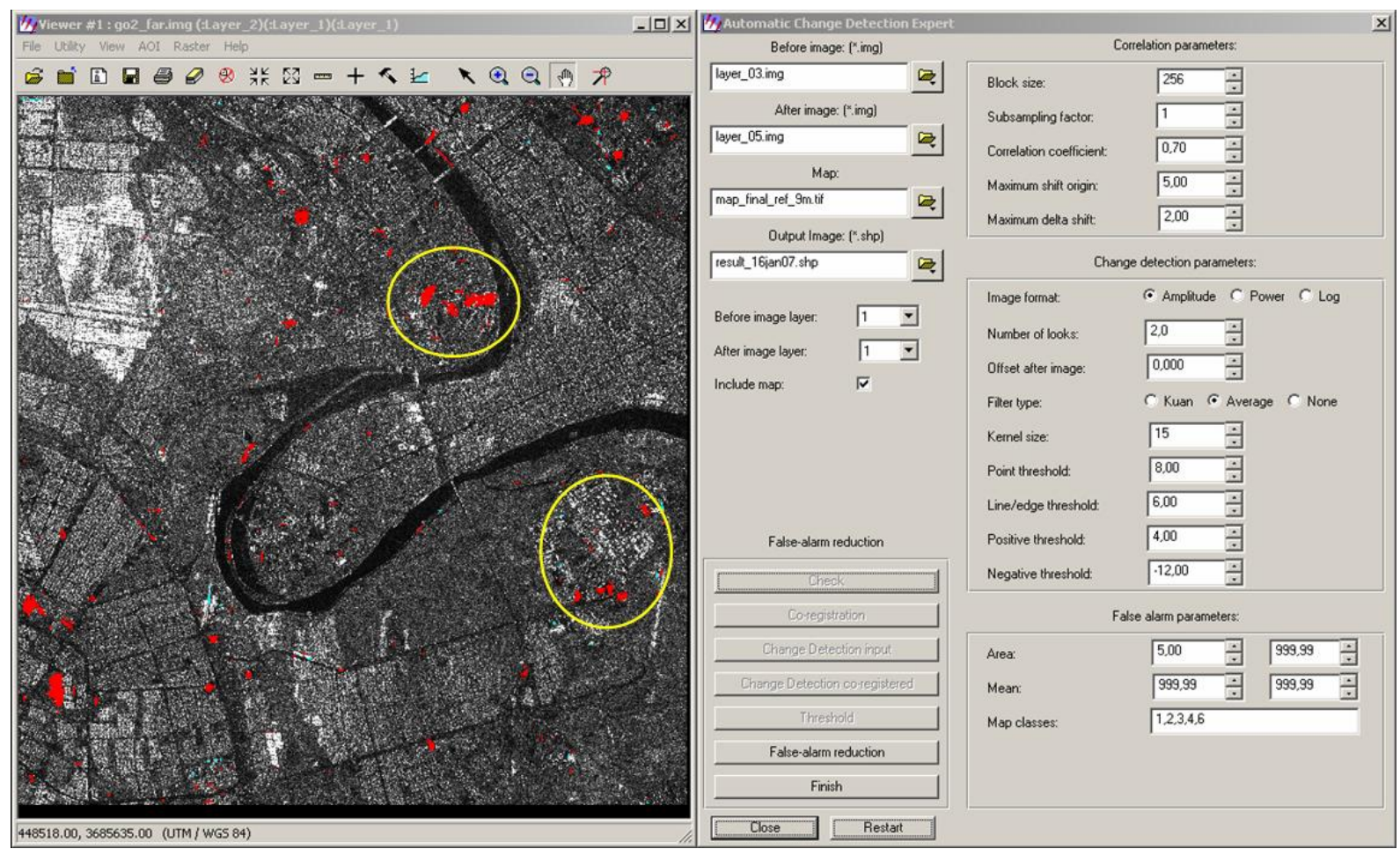

Figure 3.1. Areas of change in red overlay on a Radarsat 1 image (left) and interface of the change detection module (right).

Selected areas used in section 5 for further analysis are indicated in yellow.

We have used this change detection tool for Radarsat I fine beam data with a resolution of about 10 meters for the city of Baghdad that were collected on 17 January 2003 before Operation Iraqi Freedom in March/April 2003 and more recently on 17 July 2005. Change detection results together with the interface of the tool are shown in figure 3.1. Before the filtering images were co-registered precisely in the correlation module. We applied an average filter in the change detection procedure, which usually gives more connected areas of change and we suppressed negative changes with a high threshold. For the false alarm reduction we have used a land use map, based on a city map from the National Imagery and Mapping Agency in 2003. In this cases we have discarded changes for water using the map. We also discarded small changes with less than 5 pixels (i.e. lees than 50 meters). In section 5.1 we will classify the changes for the two areas with yellow circles indicted in figure 3.1.

\subsection{Change detection on multi-date SAR imagery}

When it is not clear at forehand when a change occurs or when dynamical variations in the scene are important multidate data-sets have to be analyzed. In such cases so-called temporal features, which indicate dynamical variation, are important. An image in a multi-date data-set is considered as a layer in a cube of images, with two spatial axes and one time axis. Successful analysis of such a set is only possible when the imaging geometry is comparable and when the images are accurately co-registered just as for a pair of images.

There are several techniques available used for multi- or hyper-spectral data sets such as principle component analysis and multivariate alteration detection (MAD, Canty, 2006), which in principle can also be used here. We describe here a technique based on an eigenvalue analysis, which we will apply to SAR data of Baghdad. 
This method consist of the following steps. 1) Subsampling. This step is done in order to reduce speckle in radar imagery and to avoid change detection effects due to inaccurate registration of the image layers. 2) Determination of main axis. In this method the intensity values of one image are assigned to one axis so that multiple images span a multidimensional space. For all the points in this space eigenvectors are calculated. The main axis is assigned to the eigenvector with the largest eigenvalue. Points which are close to this axis represent no change, but points with large distances indicate changes. The method has the advantage that it is less sensitive to an overall change of the intensity histogram, for example in case an intensity histogram is smaller or shifted, for one image compared to another image in the multi-temporal data-set. 3) Thresholding. Once the main axis has been calculated changes are detected by applying a threshold to the distance of the points to the main axis, where points with larger distances than the threshold are declared as changes. 4) Temporal features. Extraction of temporal features, which describe the changes as a function of time.

We have analysed 5 images over Baghdad from 17 January 2003, 30 March 30 2003, 23 April 2003, 17 July 2005, and 2 April 2007 which were collected with Radarsat in the same orbit, so that for all images the same imaging geometry is applicable. These so-called fine beam images have a resolution of 10 meter. The images were co-registered using a method based on correlation (Ghauharali et al., 2006) and were smoothed using a window of 5 by 5 pixels in order to be less sensitive to detect changes based on speckle. In figure 3.2 we show the Baghdad area of 25 by $25 \mathrm{~km}$ which has been analyzed. The image shows three dates in the RGB colours channels. This method is an easy way for visual inspection in order to find changes since for no change the image is grey, while a change shows up in colour. Of course when more than three dates have to be analyzed this visualization method is inadequate. In figure 3.2 various colours related to changes are visible. For example blue implies that (23 April 2003) enhanced scattering occurred just after the war, while yellow indicates the presence of enhanced scattering in 2005, which remained in 2007. Red indicates recent changes indicated by enhanced radar scattering.

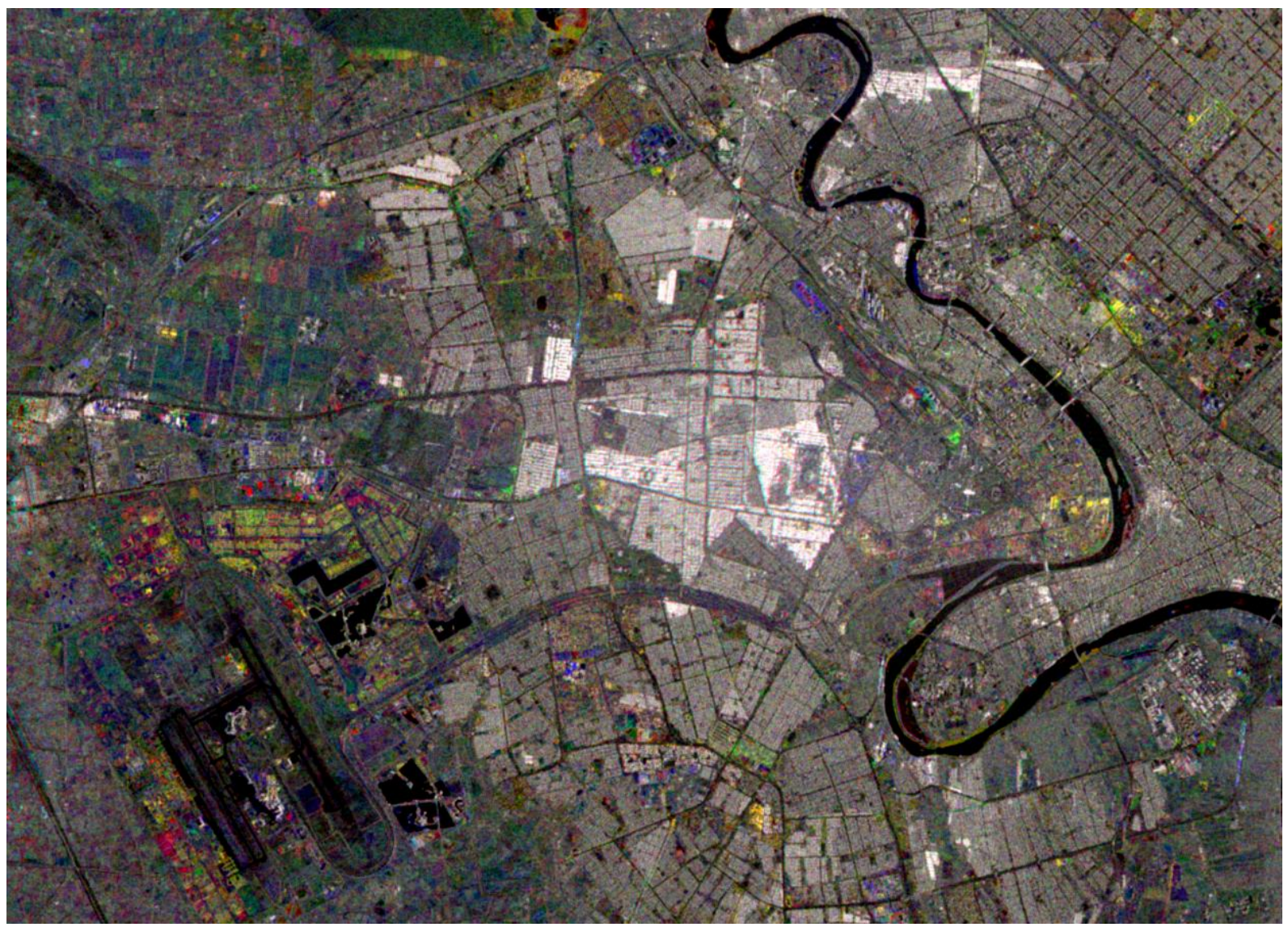

Figure 3.2. Multi-temporal Radarsat image of the Baghdad area from 23 April 2003 (blue), 17 July 2005 (yellow) and 2 April 2007 (red). 
In section 5.2 we will use multi-temporal feature to find and characterize multi-temporal changes, which we will classify with high-resolution optical imagery from other dates.

\section{CHANGE CATEgORIZATION}

We present here a workflow for categorization of the changes (van den Broek et al., 2005). The first step is to only select significant changes by removing irrelevant changes and so-called false alarms. This step reduces the number of changes to be considered and involves knowledge (features) about objects that are to be monitored, knowledge about the sensor, and combination with geographical information of the scene.

In the second step changes are positively selected and identified from the reduced set of changes, where context information (maps and optical images), information from earlier monitoring (time series) and preferences of the user are used. In the following figure we give an overview of the workflow.

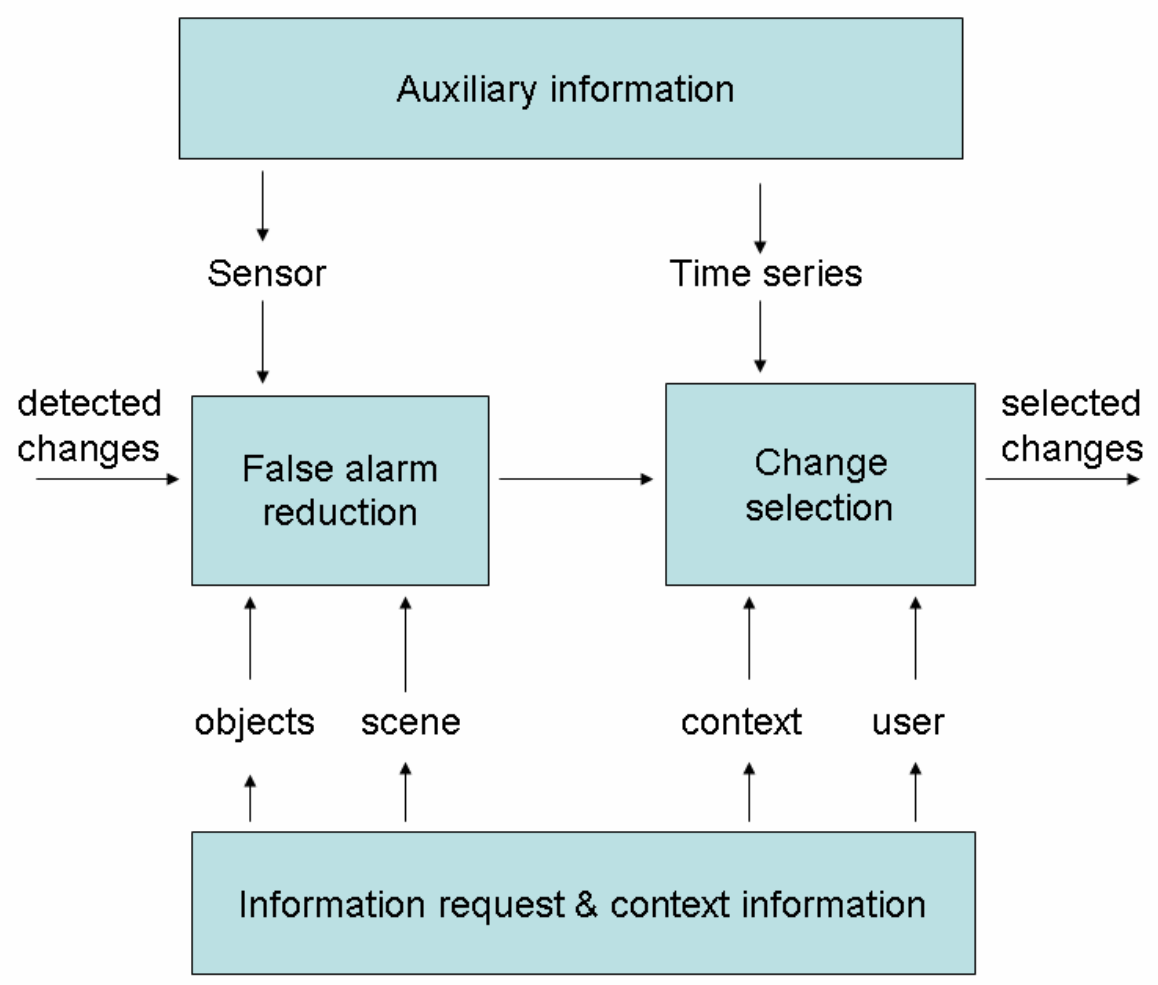

Figure 4.1. Workflow for change categorization

\subsection{False alarm reduction}

Changes in imagery can be due to various causes other than a real change on the surface. For example a change in specular reflection, or change in shadow due to little differences in aspect and incidence angles, can cause change detection. These kind of detected changes are typically related to the sensor and the sensor geometry and can clearly be discarded. If we know what we are looking for, e.g. an object with specific dimensions and specific radar signatures we can discriminate such a change from changes with other dimensions and signatures. Another way to reduce the number of changes is knowledge about the scene, when it contains areas where changes are not important. For example, in case 
of maritime surveillance only changes on water surfaces need to be considered. This kind of reduction, which uses information from the sensor, object, and scene, can be performed mostly automatically. It involves feature extraction and criteria for these features, radar characteristics of objects, and geographical analysis of the scene. The criteria are quite objective and are not very susceptible to change once an optimal working procedure is obtained. In section 3.1 we have already applied initial false alarm reduction by allowing only larger changes for certain land use classes. In section 5.1 we use context information such as expected height for buildings to select and classify the changes.

\subsection{Change selection}

A second less automatic step for classification is selection by an interpreter. Selection of changes can be performed by using context information. Such information can be areas of interest, such as pipeline corridors or certain areas with a specific ethnic population. Context information may also be derived from interpretation of a high resolution optical image. In case of regular monitoring of a certain area, which is often the case for surveillance, a comparison can be made with detections from earlier monitoring cycles, so that analysis of time series is possible. This also helps to select changes. An example is given by repetitive changes due to regular parking of vehicles, which may not be interesting for the goal of the monitoring. Another reduction is the choice of the interpreter not to consider certain changes, since he assesses that these changes will not be of interest for the user. For example the user only wants to know about military activity and is not interested in changes due to civil activities. In section 5.2 we typically use multi-temporal information (time series) to select changes which will be classified with high-resolution optical imagery.

\section{CLASSIFICATION OF CHANGES AND URBAN SITUATIONAL AWARENESS}

In this section we present the results for classifying changes obtained in section 3 based the workflow in the previous section. Using other information such as optical imagery of other and older dates results for urban situational awareness are obtained.

\subsection{Classification of changes from a pair of SAR images}

For the classification of changes, we use a digital surface model processed by Joanneum Research (Raggam et al., 2004) using Ikonos stereo imagery (van den Broek et al., 2007) for two areas with apparent changes (see figure 3.1). The first area is located in the Green Zone, the heavily guarded diplomatic/government area in Baghdad. The second area comprises a large industrial complex associated with an oil refinery in the southern part (Dawrah or Dora) of Baghdad. For each of the areas we show an Ikonos image (9 December 2005) with a resolution of 1 meter and an accompanying digital surface model from the stereo data with changes in a red overlay (figures 5.1 and 5.3).

Area 1: government area (Green Zone). This area shows significant changes between 2003 and 2005 using Radarsat. In figure 5.1 three spots with the main changes are numbered and detailed imagery of 9 December 2005 from Ikonos is shown for these spots in figure 5.2. The digital surface model is also shown in figure 5.1 and has a somewhat noisy appearance due to problems in the correlation of the stereo image pair. Typical height for the river (dark area) is about 30 meters above sea level. There are also some higher areas in the river, which are due to the correlation problem. The average ground level is 34-35 meters above sea level, and the highest buildings such as the presidential palace have heights up to 60 meters above sea level. i.e. a building height of 25 meters. Using the digital surface model, average heights for the spots have been calculated (see table 5.1). The heights in table I are for all spots a few meters above the ground level. We can use these heights as an indicator for the appearance of new buildings when the height above ground level is more than 2 meters. This is true for spot 2 and 3 . When we inspect the Ikonos images for the three spots in figure 5.2 we see that spot 1 is a parking area and spots 2 and 3 are areas where barracks have been deployed after the war in 2003. These barracks are probably in use for military accommodation. The presence of the barracks enhances the scattering in the Radarsat image of 2005. The enhanced scattering for spot 1 indicates that the parking area is used after the war and not or less before the war.

Table 5.1. Average height values for the spots in area 1

\begin{tabular}{|c|c|c|c|}
\hline Spot no. & 1 & 2 & 3 \\
\hline Average height (m) & 36.7 & 37.0 & 38.5 \\
\hline
\end{tabular}



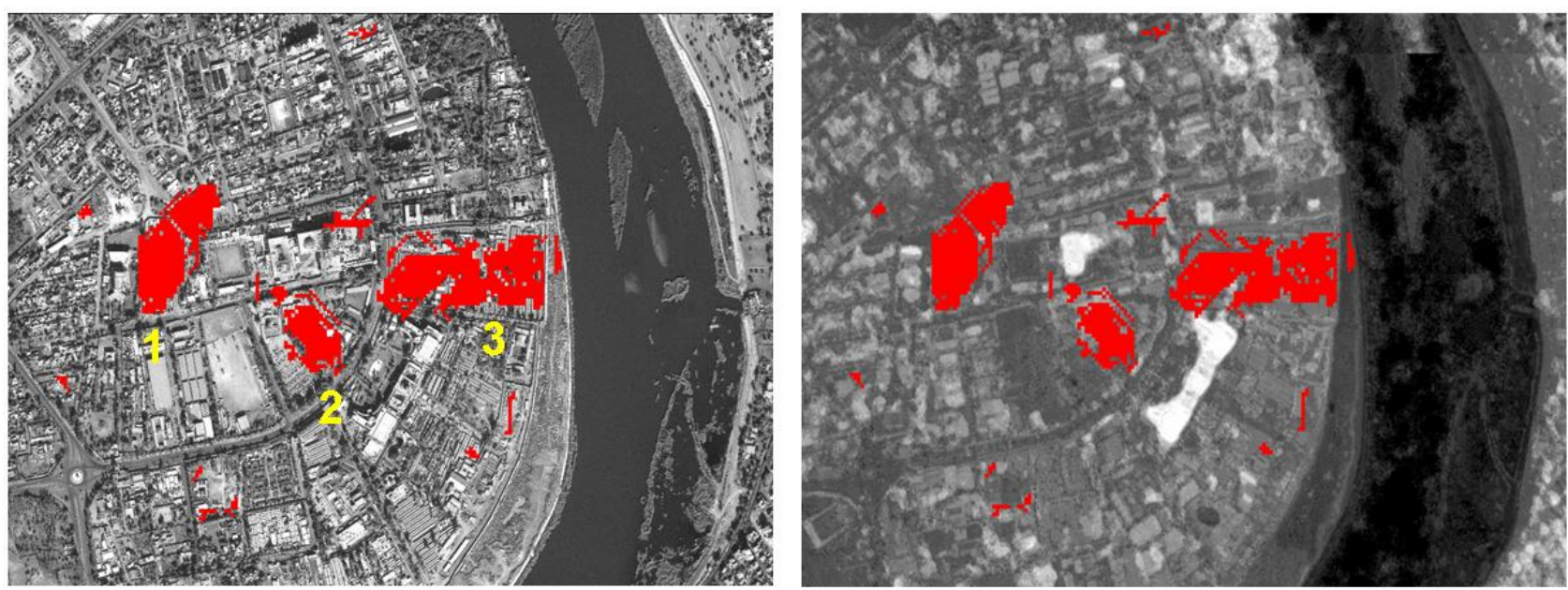

Figure 5.1. (Left) Ikonos image of 9 December 2005, with changes from Radarsat in a red overlay for area 1 (Green Zone). (Right) DSM of the same area.
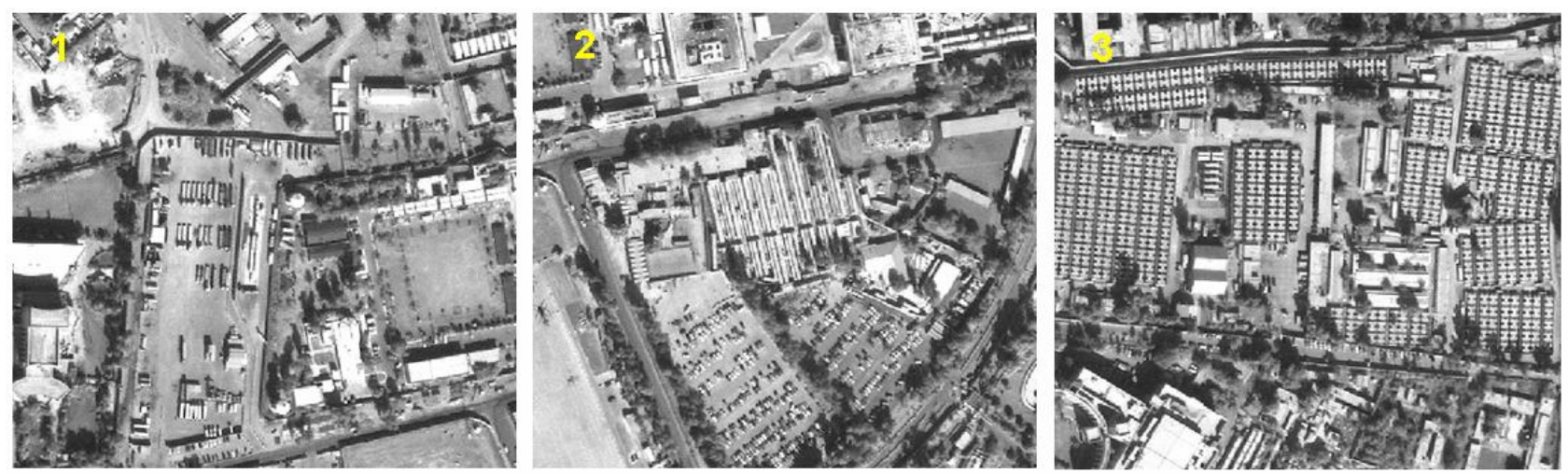

Figure 5.2. Enlargements of Ikonos image for three spots with changes in Figure 5.1

Area 2: industrial complex (Dora Refinery). For this area four spots have been selected with large changes detected using the Radarsat imagery. These spots are numbered (see figure 5.3) and can be inspected using detailed imagery of 9 December 2005 from Ikonos (see figure 5.4). Also shown is the digital surface model in figure 5.3. Height levels range from about 25 meters above sea level (dark area) to about 50 meters for the highest points. The white area to the left in the digital surface model is a high building with a height of 52 meters above sea level. Since the average ground level is in the order of 32-33 meters above sea level, the building height is about 20 meters. Other white patches are forest, with heights in the order of 45 meters above sea level, so that tree heights are on average about 12 meters. Analogously to area 1 average heights have been calculated for the four spots (see table 5.2). The heights for the spots range from 33 meters to 35 meters from which we conclude that the changes are not due to the presence of new infrastructure consisting of buildings, since this would require heights of several meters above ground level. When we inspect the Ikonos images in figure 5.4 we see that spots 1,2,3 are places where trucks are parked in assembly places. Spot 4 shows storage of goods.

Table 5.2. Average height values for the spots in area 2

\begin{tabular}{|c|c|c|c|c|}
\hline Spot no. & 1 & 2 & 3 & 4 \\
\hline Average height $(\mathrm{m})$ & 35.1 & 34.1 & 33.1 & 33.4 \\
\hline
\end{tabular}



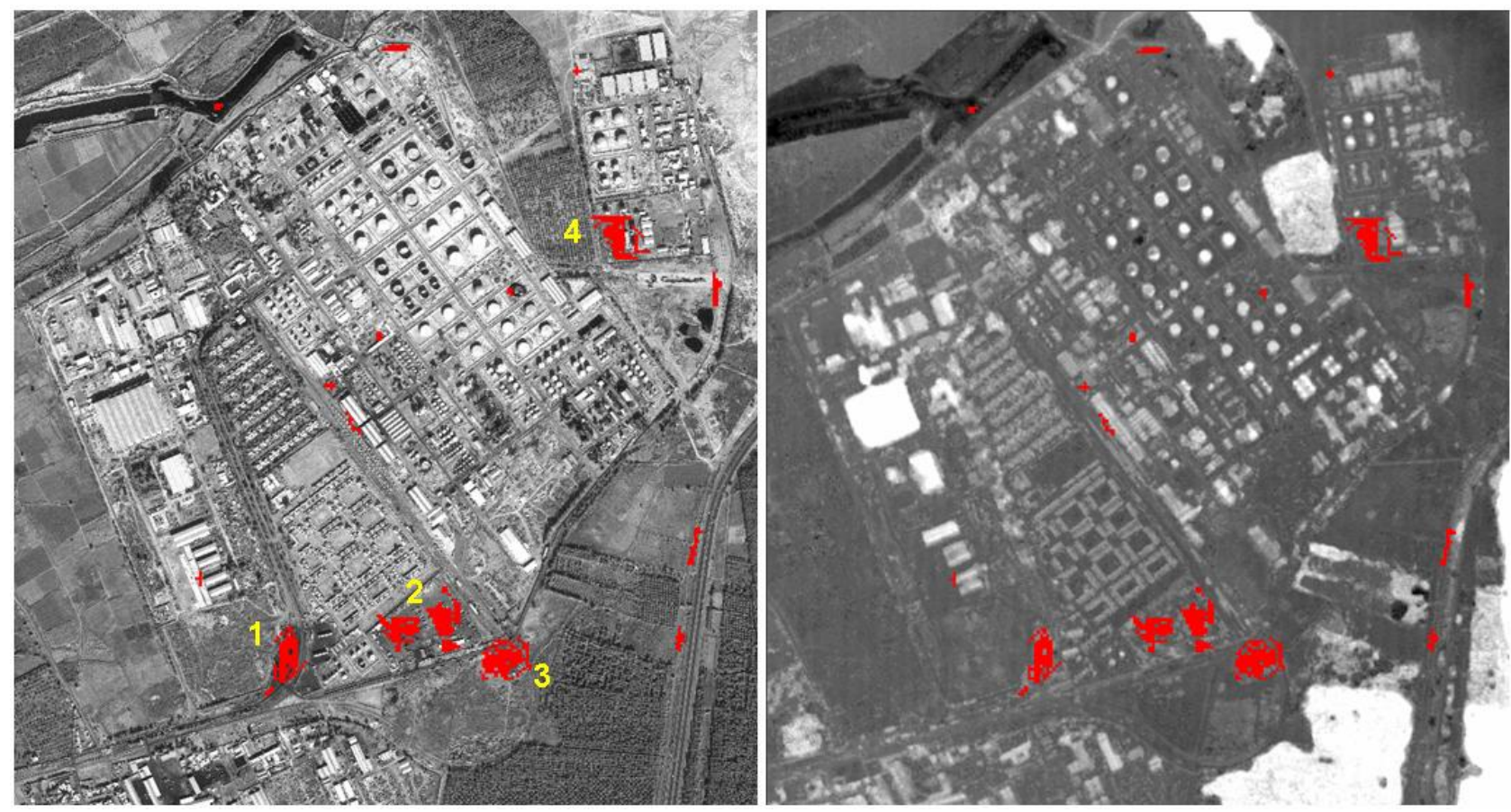

Figure 5.3. (Left) Ikonos image of 9 December 2005, with changes from Radarsat in a red overlay for area 2 (Refinery).

(Right) DSM of the same area.
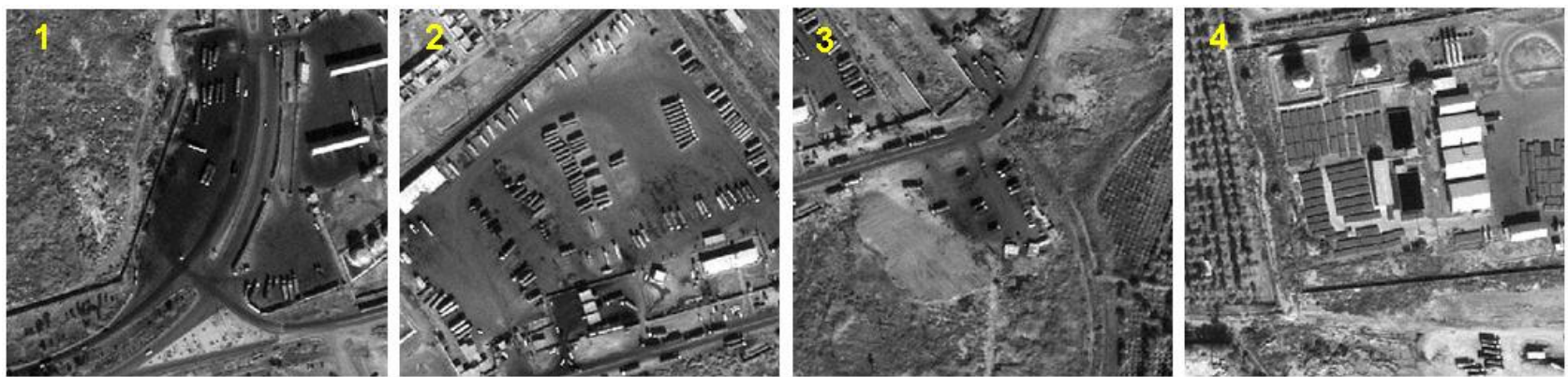

Figure 5.4. Enlargements of the Ikonos image for four spots with large changes in figure 5.3.

These assembly places for trucks are quite common in large cities such as Baghdad. They are usually found near large roads and crossing points. When trucks are present the scattering is increased so that apparently more trucks are found at these assembly places in 2005 compared to the period before the war in 2003. Presence of goods stored at spot 4 in 2005, but absent in 2003 explains the increase in the scattering. On basis of these observations we can conclude that activities near the refinery have increased after the war which is in agreement with the restrictions due to the economical sanctions for Iraq before the war in 2003. The restrictions also imposed a low activity in the oil sector.

\subsection{Classification of changes from multi-date SAR imagery}

In this case the multi-temporal behavior can serve as information for characterizing the changes. In section 3.2 we have described and applied the multi-temporal change detection method to the Radarsat data-set consisting of the five dates available. The detected changes have been analyzed for temporal behaviour, which is described by so-called temporal features. We define temporal features (TF) which elements can have values $-1,0$, and +1 , where " 0 " means no change, " +1 " means the scattering increased and " -1 " means the scattering decreased between two dates. In principle for a multitemporal data-set of $N$ layers there can be $(N-1)^{3}-1$ possible temporal features. We discuss here 4 temporal features, which are summarized in table 4.3. Feature 1 refers to enhanced scattering in the second phase of the war, decrease after 
the war and again increase during the recent years. Feature 2 refers to enhanced scattering before and after the war and a decrease during the last two years. Feature 3 refers to enhanced scattering after the war with ongoing increase in the last two years. Feature 4 refers to enhanced scattering after the war in 2005, which disappeared in 2007 again.

Table 5.3. Temporal features (TF) used in the analysis and colours used in figure 5.5

\begin{tabular}{|l|l|l|l|l|l|}
\hline & $\begin{array}{l}\text { 23jan03- } \\
\text { 30mar03 }\end{array}$ & $\begin{array}{l}30 \mathrm{mar} 03- \\
23 \mathrm{apr} 03\end{array}$ & $\begin{array}{l}23 \mathrm{apr} 03- \\
17 \mathrm{jul05}\end{array}$ & 17jul05-2apr07 & colour \\
\hline TF 1 & 0 & +1 & -1 & +1 & red \\
\hline TF 2 & -1 & 0 & +1 & -1 & green \\
\hline TF 3 & 0 & 0 & +1 & +1 & cyan \\
\hline TF4 & 0 & 0 & +1 & -1 & purple \\
\hline
\end{tabular}

In figure 5.5 a map of Baghdad is shown with in overlay 4 areas with Radarsat data of 23 April 2003. For these areas changes are selected using one of the temporal features shown in colours as given in table 5.3).

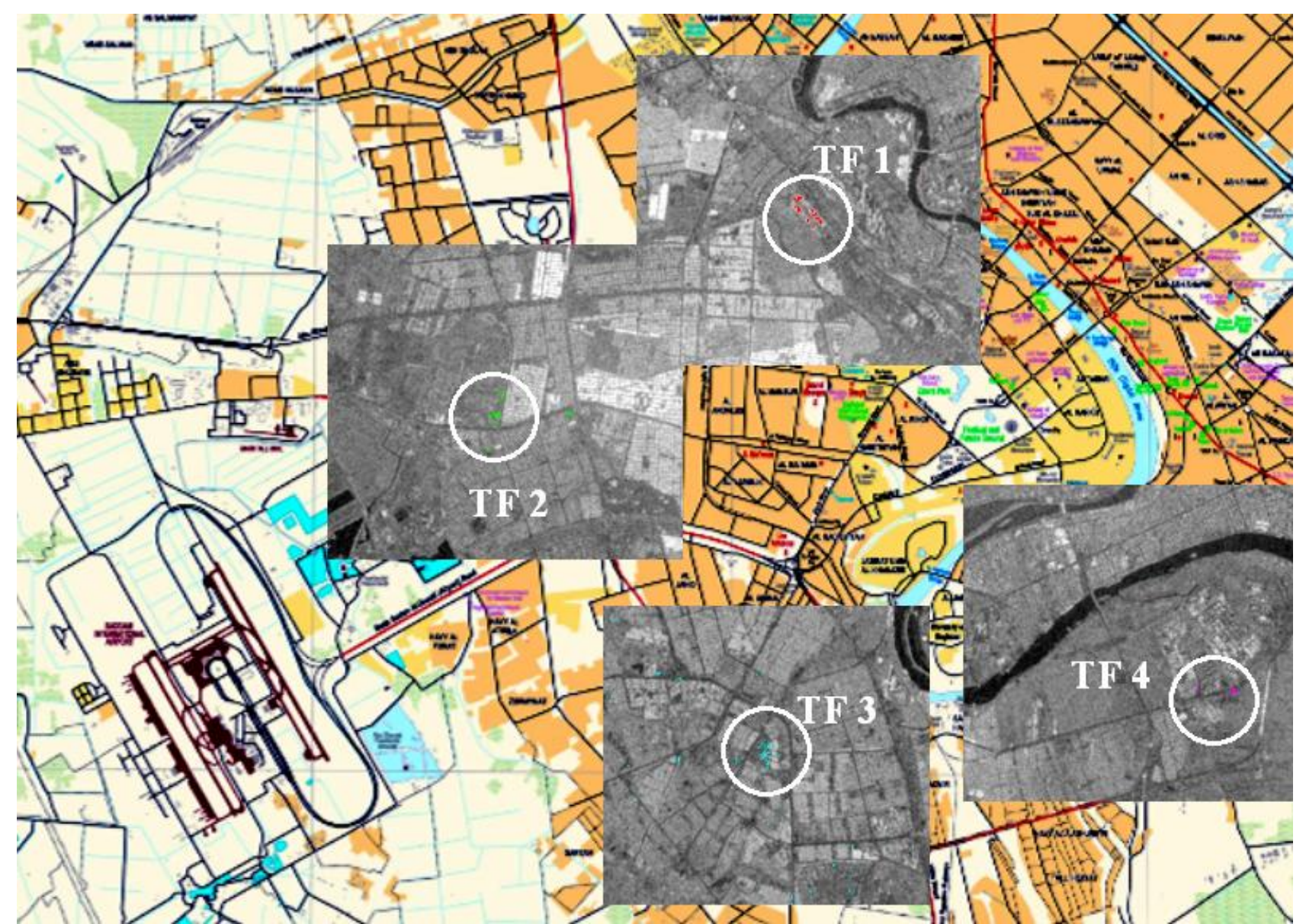

Figure 5.5. Map of the same area as shown by the Radarsat image in figure 3.2. Map has been produced by NGA and has been made available to the public on internet (NGA stock number: K942SBAGHDAD).

The circles indicate the selected changes which have been studied in more detail, by comparing high resolution optical imagery of before, during and after the war of the same spot (see figs. 5.6-5.8). We discuss the examples for the 4 features in more detail. 
Temporal feature 1 refers to enhanced scattering in the second phase of the war. A possibility is destruction of e.g. (military) storage barracks. Debris from such barracks will give enhanced and more distributed scattering compared to scattering from non-destructed barracks. Later on the area will has been be cleared. Several cases have been found in Baghdad. The selected area (figure 5.5) is shown in Quickbird imagery of 1 April 2003, of 9 April 2003, and compared with satellite imagery from Google Earth, estimated to be from 2005 (figure 5.6). From figure 5.6 we see that destruction took place between 1 and 9 April and that the area has been cleared in 2005. Apparently in the last 2 years this area has been reused again and new infrastructure has been build or equipment has been stored, which gives rises to enhanced scattering again.
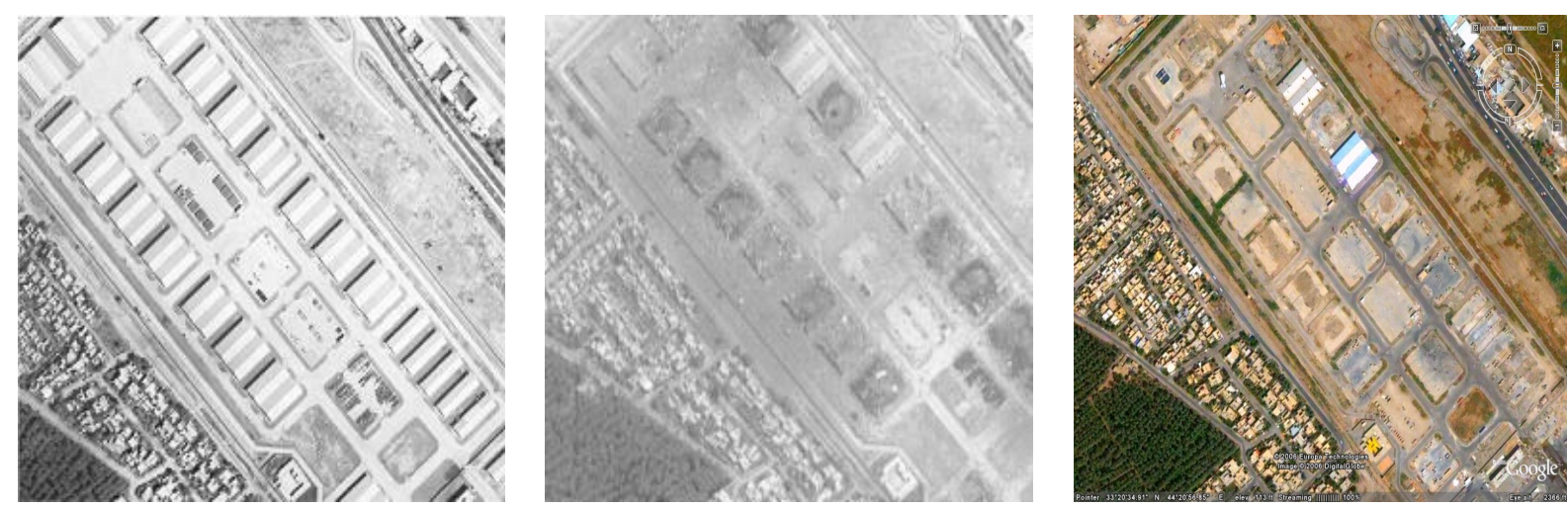

Figure 5.6. Area of about 800 meter corresponding to a detected change with TF1. The area is shown in Quickbird imagery of 1 April 2003, of 9 April 2003 (middle) and Google Earth imagery of 2005 (estimated, right).

Temporal feature 2 refers to enhanced scattering before and after the war with a decrease in scattering during the war. This can be expected for trade centers and market places. In such areas usually many trucks are present, which can be observed by Radarsat due to enhanced scattering. However during the war trucks were removed and hidden for protection, so that the areas were more desolated. Such centers are often found near crossings of main roads. For the selected area (figure 5.5) we show an Ikonos imagery of 2000, a Quickbird image of 9 April and a Google Earth image of 2005 in figure 5.7. These images show that indeed less trucks were present during the war. Apparently during the last two years the area has fallen into disuse, possibly due to governmental regulations or due to the worsening security situation.
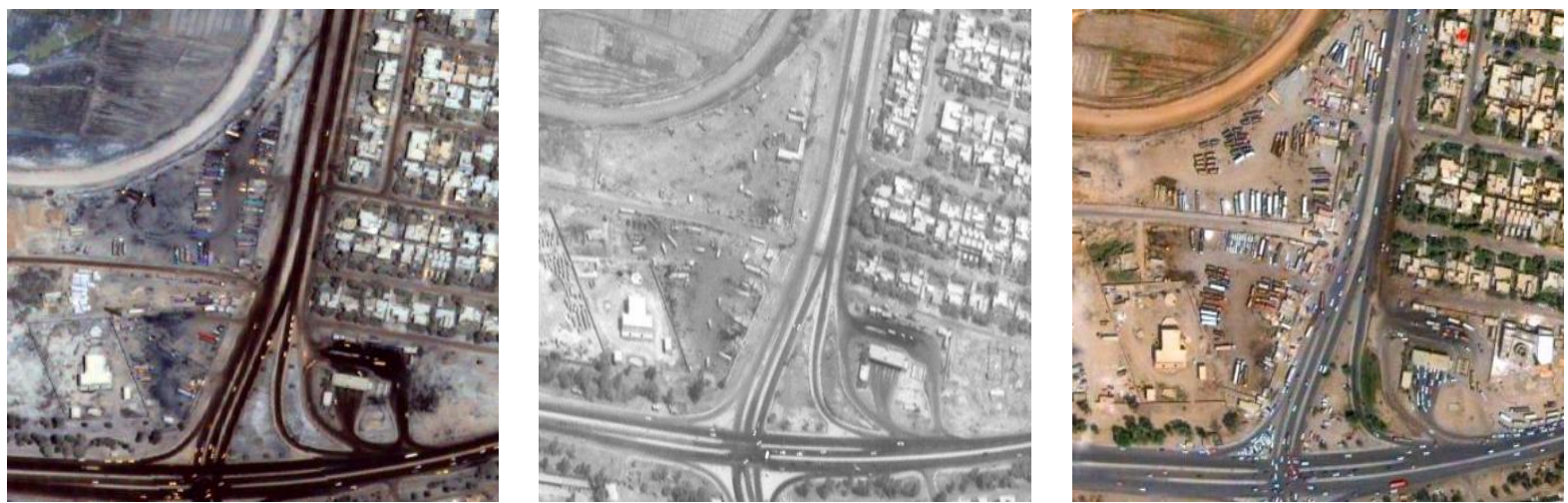

Figure 5.7. Area of about 400 meter corresponding to a detected change with TF2. The area is shown in Ikonos imagery of 2000 (left), Quickbird imagery of 9 April 2003 (middle) and Google Earth imagery of 2005 (estimated, right).

Temporal feature 3 refers to enhanced scattering only after the war, which is increasing during the last two years. This typically can be expected for new infrastructure built after the war on empty places. For the selected area (figure 5.5) we 
show an Ikonos imagery of 2000 and a Google Earth image of 2005 in figure 5.8. The figure shows that on agricultural land in 2000 various dwellings have been built in 2005. Apparently the building of such dwellings went on between 2005 and 2007
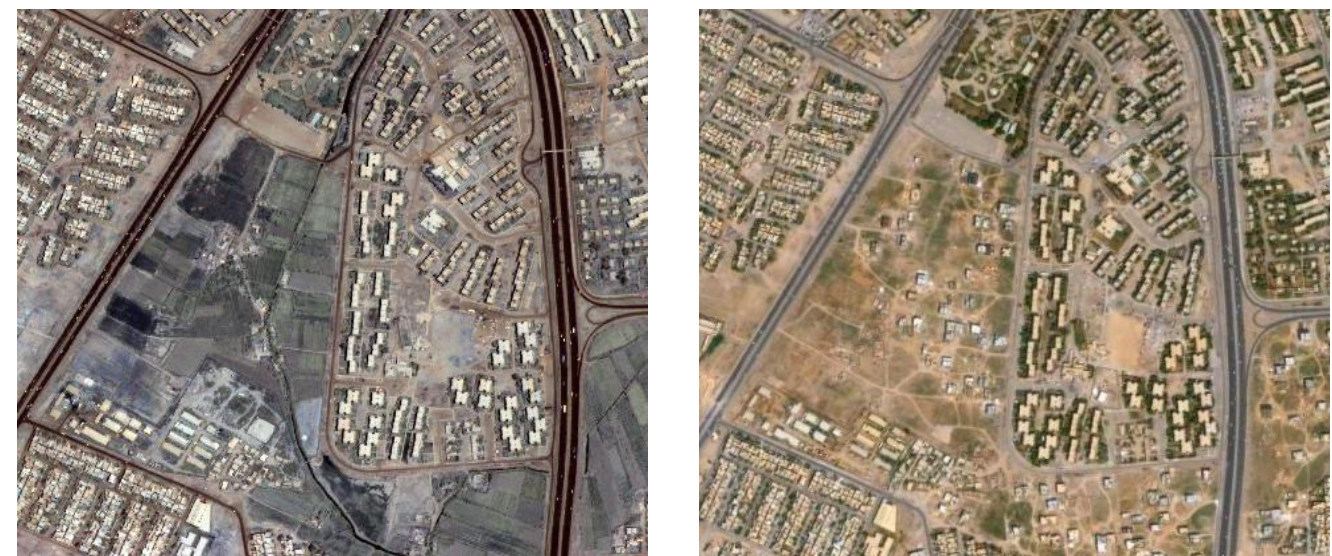

Figure 5.8. Area of about 800 meter corresponding to a detected change with TF3. The area is shown in Ikonos imagery of 2000 (left), and Google Earth imagery of 2005 (estimated, right).

Temporal feature 4 refers to enhanced scattering after the war in 2005 that has disappeared in 2007. The area found is the same area near the Dorah Refinery discussed in section 5.1. The changes found coincide with spots 1 and 3, for which it was concluded that trucks were present in 2005. Apparently these spots have been cleared in 2007.

\section{SUMMARY AND CONCLUSIONS}

We have proposed a workflow for regular monitoring and surveillance with SAR in combination with high-resolution optical imagery and other information such as geographical information. SAR data is well suited for change detection, but does not give sufficient information to classify these changes. We have proposed 2 steps to handle the detected changes. Step 1 involves reduction of irrelevant changes, so-called false alarms. Step 2 comprises selection of the remaining changes by an interpreter. In these steps information about the object, sensor, scene, time series, user and context are important. The optical data, and other geographical information are then used to classify these selected changes.

We have applied change detection techniques to Radarsat I fine beam imagery to find changes in Baghdad during and after the war in 2003. Taking into account above-mentioned workflow we have used Ikonos stereo imagery and a digital surface model that contains building heights to classify the changes. We have also used so-called temporal features extracted from a multi-temporal data-set of Radarsat data to select the changes and to detect activity between 2003 and 2007, which has been classified with high resolution optical data.

Although the described topics are clearly significant contributions to urban situational awareness, they are only part of it. Other issues such as information about areas with different ethnic populations, or about areas with a high risk for terrorist attacks are also important contributions to urban situational awareness.

\section{REFERENCES}

1. Canty, M.J., 2006, Image Analysis, classification and change detection in remote sensing, Taylor \& Francis, ISBN0-8493-7251-8.

2. Dekker, R.J., 2005, SAR change detection techniques and applications, 25th EARSeL Symposium on Global Developments in Environmental Earth Observation from Space, 6-11 June 2005, Porto, Portugal. 
3. Ghauharali, R.I., Dekker, R.J., van den Broek, A.C., van Maarseveen, R.A., 2006, Automated detection and categorisation of changes in satellite imagery, NIVR report 53501VB, August 2006.

4. Raggam, H., Franke, M., Gutjahr, K, 2004, Surface Model Extraction from High Resolution Stereo Data over Alpine Terrain, 24th Earsel Symposium "New Strategies for European Remote Sensing, Dubrovnik, May 25 - 27, 2004.

5. Van den Broek, A.C., Dekker, R.J., and Steeghs, T.P.H., 2005, Concepts for monitoring and surveillance using space borne SAR systems, NATO-SCI-150 symposium on Integration of Space-based assets within full spectrum operations, 10-12 October 2005, Colorado Springs, USA.

6. Van den Broek, A.C., Dekker R.J, Gutjahr, K., Raggam, H., 2007, Use of high resolution optical and radar imagery for intelligence and situational awareness in urban areas, proceedings Urban Remote Sensing Joint Event, Paris 1113 April 2007. 